\title{
Variations
}

Variations

Revue internationale de théorie critique

$17 \mid 2012$

Critique du travail

\section{Dans le labyrinthe du Minotaure}

critique du travail. Le faire et l'agir, 25 ans après

\section{Alexander Neumann}

\section{(2) OpenEdition}

Journals

Édition électronique

URL : http://journals.openedition.org/variations/432

DOI : 10.4000 /variations. 432

ISSN : 1968-3960

Éditeur

Les amis de Variations

Référence électronique

Alexander Neumann, "Dans le labyrinthe du Minotaure », Variations [En ligne], 17 | 2012, mis en ligne le 15 octobre 2012, consulté le 01 mai 2019. URL : http://journals.openedition.org/variations/432 ; DOI : 10.4000/variations.432

Ce document a été généré automatiquement le 1 mai 2019.

Les ami•e•s de Variations 


\title{
Dans le labyrinthe du Minotaure
}

\author{
Critique du travail. Le faire et l'agir, 25 ans après
}

\section{Alexander Neumann}

1 La valeur travail pullule dans l'espace public bourgeois, alors que l'Europe est au chômage et que parmi les salariés les plus sollicités se trouvent celles et ceux qui se réfugient dans le suicide. Que faire? La question léniniste est à la fois simple et désuète, puisqu'elle invite au raccourci activiste, à la fainéantise intellectuelle et à l'utilitarisme. Comment penser une politique de l'émancipation qui ne soit pas une simple répétition des schèmes de mobilisation dont se servent le management, les mass media, les institutions qui veulent conjurer la crise? Les belles pages de Jean-Marie Vincent qui jaillissent dans Critique du travail. Le faire et l'agir, furent publiées pour la première fois en 1987, il y a 25 ans, aux PUF. Variations, revue dont l'auteur fut le fondateur, réédite aujourd'hui ce livre ${ }^{1}$ recherché mais introuvable avec l'aimable accord d'Isabelle Vincent, ainsi que le dialogue critique entre André Gorz et Jean-Marie Vincent entamée lors de la sortie du premier numéro de Variations, en 2001. Plusieurs contributions viennent d'en indiquer la pertinence et les soubassements, lors que d'autres explorent la critique du capitalisme chez Théodor W. Adorno à laquelle ce débat se réfère. ${ }^{2}$

2 Critique du travail se situe à la croisée des chemins, entre la fin d'un monde et la quête d'un nouveau souffle critique. La gauche est alors en train de péricliter. Le gouvernement socialiste vient d'organiser le «tournant de la rigueur » sous Mitterrand, tandis que le communisme stalinien livre une dernière parade triste avant la chute du Mur de Berlin en 1989. Sur le plan intellectuel, nombre de sociologues venues de la gauche critique épousent le tournant culturaliste qui évacue la critique du capitalisme de la scène théorique (Alain Touraine, Jürgen Habermas), quand ils ne se livrent pas à des confusions idéologiques sous l'égide des nouveaux philosophes, qui ont fini en vieux sarkozystes (André Glucksmann, Alain Finkielkraut). Tout devait disparaître, les utopies, le prolétariat, la critique et même l'art contemporain, emportés par la mort des grands récits.

Les lignes que l'on va lire [...] ne veulent pas se situer dans les courants dominants à

l'heure actuelle, si souvent marquées par la passion réactionelle et réactionnaire 
face au passé récent, ou encore si marquées par la résignation désabusée devant les

faits accomplis du présent et de la marche folle des sociétés actuelles. avec les concepts de Marx est alors un acte gratuit, un acte de résistance. Jean-Marie Vincent disposait de l'obstination nécessaire, matinée de rigidité pour ceux qui ne le connaissaient pas de près, pour faire face à l'éboulement de terrain politique et mental de ces heures noires. Il avait une grande culture historique qui lui a permis de cerner l'arrivée de Mai 68 , le déclin de l'Etat social hérité de l'après-guerre et l'épuisement du socialisme bureaucratique. Il a surtout bénéficié d'une double culture germanophone et francophone, venue de sa jeunesse lorraine, qui l'a préservé du nombrilisme universitaire parisien. Sa thèse à l'Institut de sciences politiques de Paris sous la direction d'Alfred Grosser avait porté sur la formation d'une nouvelle gauche en Allemagne de l'Ouest pendant la Guerre froide. ${ }^{3}$ Les expériences vivantes d'un marxisme critique lui ont permis de recevoir la Théorie critique de l'Ecole de Francfort.

Pour ces raisons, le livre ne resta pas un baroud d'honneur, mais inaugura un espace de discussion aux côtés de Toni Negri, André Gorz, Michael Löwy, de féministes comme Jacqueline Heinen et d'autres universitaires, attachés à l'esprit de l'Université de Vincennes. Le département de sciences politiques dirigé par Jean-Marie Vincent vit ainsi naître la revue Futur antérieur (sous sa direction) qui anima les débats de la gauche critique jusqu'en 1999, date de l'essor du mouvement altermondialiste à Seattle. À partir de 2001, Vincent engagea à travers Variations la prolongation de cette expérience d'un Futur antérieur, ce moment qui adviendra et qui aura existé pour les générations futures, à la manière d'une utopie concrète, toujours agissante. Critique $d u$ travail préfigure cet étonnant et fructueux brassage des cultures intellectuelles de gauche. Le livre s'inspire de l'engagement existentialiste venu de Gorz, d'une lecture marxienne révolutionnaire venue des Grundrisse et d'emprunts à la Théorie critique, notamment à travers une réception critique de l'œuvre d'Ernst Bloch et des incursions dans la théorie de Jürgen Habermas. Le livre se compose de cinq essais, dont la forme même signale une subjectivité rebelle à l'encontre de toute pensée schématique ou administrée, à travers un flux et un raffinement de l'écriture qui se situe à l'opposé de l'industrie du spectacle. L'auteur ne veut pas se situer dans les courants dominants et refuse de vivre ou penser comme des porcs. L'arc de tension de l'ouvrage part d'une déconstruction magistrale des fondements du marxisme léniniste chez Lukács, passe par une relance de la critique du fétichisme chez Marx et arrive à une mise en perspective de cet héritage en faveur d'une auto-transformation de la société pour finir en point d'orgue sur une autre conception de la révolution. Les chapitres deux et trois portent sur la philosophie de l'utopie chez Bloch et une discussion d'Heidegger à partir de Marx. Si la conclusion du chapitre sur Heidegger, la politique révolutionnaire dans l'élément de la finitude, sonne comme une anticipation de la pensée de la décroissance, la référence à Heidegger est sans doute le point le moins exploré de l'argumentation de Vincent. Ici, Vincent se situe pleinement dans la tradition française d'une appropriation philosophique de l'existentialisme dans la lignée de Sartre et de Gorz, alors que la critique fondamentale qu'Adorno, Marcuse et Habermas adressent à Heidegger restent en retrait de l'argumentation. Si Marcuse avait déjà rompu avec le penseur antisémite depuis la guerre, à travers une lettre cinglante, alors qu'Adorno avait à juste titre moqué le discours identitaire d'Heidegger dans le Jargon de l'authenticité, ${ }^{4}$ l'ampleur de l'engagement nazi n'est cependant apparu qu'après la publication du livre de Vincent. Victor Farias a montré la continuité des convictions fascistes de l'homme 
Heidegger jusqu'à ses derniers jours, tandis qu'Emmanuel Faye a fidèlement retracé l'imbrication problématique des concepts politiques et philosophiques d'Heidegger dans L'introduction du nazisme dans la philosophie. ${ }^{5}$ Du point de vue de l'élan discursif de JeanMarie Vincent, qui n'entend nullement banaliser des concepts réactionnels ou réactionnaires, le cas Heidegger pose néanmoins problème. Car le philosophe existentialiste cherche à dissoudre la dialectique, comme Emmanuel Lévinas l'a bien saisi, là où Vincent veut la remettre en mouvement en faveur d'une pensée révolutionnaire.

5 L'apport fondamental de Jean-Marie Vincent se situe sur un autre plan, celui de la réception francophone et postmarxiste de l'Ecole de Francfort et de sa compréhension de la critique du capitalisme, puis de ses formes sociales. ${ }^{6}$ Plus précisément, il tourne les arguments de Sohn-Rethel et d'Adorno contre une certaine banalisation de la critique du salariat, au moment où Habermas tourne le dos à toute critique de l'économie politique et que le marxisme doctrinaire s'effondre sur lui-même. Vincent comprend alors le capital en tant qu'une totalité abstraite, à travers des concepts tels le fétichisme de la marchandise et la soumission réelle du salariat. Concepts marxiens qui se trouvent déjà soulignés par Adorno dans un article au sujet de « Marx et les catégories fondamentales de la sociologie ". ${ }^{7}$ La contribution sociologique décisive d'Adorno, en introduction du congrès de sociologie à Francfort en 1968, avait déconstruit la notion de société industrielle avec les mots de la critique de l'économie politique. Dans le titre et dans le texte, Adorno oppose le paradigme de la société industrielle et de sa production matérielle qu'il juge peu pertinent, à la critique du capitalisme tardif dont il esquisse les principes d'organisation. En 1972, Habermas reprend encore explicitement cette approche critique dans son commentaire des Problèmes de légitimation du capitalisme tardif. À l'origine, Adorno déconstruit le discours marxiste avec et contre Marx, pour mieux mettre à l'épreuve le paradigme de la société industrielle.

Dans un essai fondamental de près de 50 pages $^{8}$, au sujet des problèmes catégoriels d'une théorie critique de la société, Axel Honneth complète la redéfinition programmatique que propose Habermas à partir de 1980, afin de justifier l'expulsion de la critique du travail et de la critique de l'économie politique de leur champ sémantique et conceptuel. L'ouvrage collectif dans lequel paraît l'essai, dirigé par Honneth en personne, cherchait à accompagner le passage conceptuel de la critique de l'économie politique - d'inspiration marxienne ou adornienne - vers le tryptique Travail, Agir, Normativité qui opère un déplacement sémantique. Ce projet d'une redéfinition habermassienne de la Théorie critique est explicitement nommé par Honneth ${ }^{9}$, au terme d'une longue déconstruction de la critique marxienne et marxiste du capitalisme, et qui cherche à neutraliser cette influence théorique au sein de l'Ecole de Francfort sans en nommer les représentants, notamment Oskar Negt et Gerhard Brandt. Les thèses marxiennes du fétichisme de la marchandise et de la soumission réelle, actualisées par les auteurs de l'Ecole de Francfort, sont contournées. Au centre de la critique honnethienne se trouvent les écrits philosophiques de Marx, puis le marxisme français d'Althusser et de Sartre. L'argumentation de 1980 peut paraître anachronique aujourd'hui, au moment où la critique de l'économie est actualisée sous la forme de best-sellers et où l'éditeur allemande du Capital de Marx est en rupture de stock ${ }^{10}$, dans la mesure où Honneth postule d'abord que la critique marxienne du capitalisme " ne peut plus rien apporter » à l'analyse des crises et conflits qui traversent les sociétés contemporaines. ${ }^{11}$ Honneth luimême considère, en 2011, que la crise mondiale actuelle du capitalisme peut être comparée à celle ouverte en 1929, même si aujourd'hui « l'intervention étatique en faveur 
des conditions de la valorisation capitaliste échappe au regard public» en raison du niveau d'abstraction du procès de valorisation dont il est question..$^{12}$ C'est précisément la critique du travail abstrait, du fétichisme et de la soumission réelle qu'il avait tenté d'abolir auparavant. En 1980, l'auteur passe d'une démonstration de l'insignifiance sociologique du concept de travail abstrait chez Marx à la sociologie du travail qui a pour objet la rationalisation taylorienne (thème qui le rapproche de Weber), et enfin au concept d'agir instrumental. Celui-ci a pour corollaire le concept d'agir communicationnel, qui définit le nouveau paradigme habermassien. Alors que dans cette optique, le travail abstrait et l'abstraction marchande semblent jouer aucun rôle dans la production des significations sociales, réservées à l'agir communicationnel, Honneth a évolué vers une position plus nuancée. Il tente ainsi de réduire la coupure qu'il a luimême contribué à mettre en place en 1981, entre travail et espace public, en regrettant que la manière dont le travail salarié se distribue au sein de la société ne joue aucun rôle dans le cadre de la théorie l'agir communicationnel. ${ }^{13}$ Nancy Fraser a jugé sévèrement cette limitation habermassienne-honethienne de la critique de l'économie politique à un "paradigme de la production» qui aurait été dépassé par un "paradigme de la communication » et de la reconnaissance : «Au lieu de dépasser l'économisme en faveur d'une théorie plus riche (Honneth) a remplacé un paradigme incomplet par un autre, celui de l'économisme contre un culturalisme limité. ${ }^{14}$ De même, le tournant habermassien de 1981 est commenté par Negt de manière critique : «Rétrospectivement, les thèses habermassiennes qui postulent l'épuisement des théories du salariat ainsi que la dissociation du système et du monde vécu, apparaissent comme des diagnostiques déplacés $\gg \cdot{ }^{15} \mathrm{La}$ critique vise en premier lieu la séparation radicale, chez Habermas, du travail et de l'action, du salariat et de l'espace public, ou encore du système et du monde vécu, comme si ces dimensions n'interagissaient pas au sein d'une même société mais concernaient deux planètes distinctes.

7 Jean-Marie Vincent suit une autre voie, cherche un autre Marx, prend Habermas à rebrousse-poil, avec Adorno et Sohn-Rethel. Ces théoriciens précisent le caractère inédit de la totalisation sociale du capitalisme, qui mêle la fausse représentation à la réalité objectivée, quand ils comparent la condition salariale au fétichisme de la marchandise. Celui-ci efface le caractère social de la production au profit de la circulation de marchandises anonymes, interchangeables sur le marché, et qui paraissent contenir une valeur particulière, alors que c'est le travail social qui leur a conféré leur valeur et leur utilité. Adorno constate : "Même si nous sommes en mesure de percer la fausse apparence de la société, cela ne modifie pas l'aspect fétichiste de la marchandise : tout commerçant qui calcule ses affaires est obligé de se comporter d'une manière conforme à ce fétiche. S'il ne le fait pas, il coule. » ${ }^{16}$ De même, si les salariés veulent vivre de leur force de travail, ils doivent accepter d'être rémunéré sous la forme monétaire du salaire, qui fait partie de la représentation abstraite du capitalisme. La valorisation des marchandises quitte ainsi la problématique de l'exploitation et de l'aliénation du producteur, pour se présenter comme une contrainte sociale "universelle». Jean-Marie Vincent présente les caractéristiques de cette contrainte : «se vendre, se faire apprécier en tant que porteur de force de travail mesurable, faire entrer les autres dans des activités de valorisation, développer des activités stratégiques de valorisation en leur subordonnant les autres activités (notamment affectives)... ». ${ }^{17}$

8 Loin de ne constituer qu'une perception illusoire de la réalité, les représentations abstraites décrivent une forme ultime de la réalité sociale, en signant la priorité des marchandises objectivées devant les relations humaines. Le renversement historique qui 
s'est opéré dans les relations sociales se répercute ainsi dans le changement des catégories de la réalité. Marx parle à ce propos de "formes de pensée objectives", puisque la pensée doit tenir compte de l'objectivité de la situation sociale. ${ }^{18}$ Dans cette optique, l'analyse critique du capitalisme, menée par Marx, ouvre la voie à une critique sociologique des formes sociales et des pratiques. L'enchantement devant le pouvoir suggestif de la marchandise, les représentations fétichistes, le mode de consommation et les attitudes individuelles, possessives, qui se fondent sur la soumission réelle du salariat, coiffent en grande partie la vie sociale. Vincent résume : «La vie des individus est marquée par la logique de valorisation, et les formes de vie (le vécu, le quotidien) s'adaptent aux formes de la valeur, c'est-à-dire aux choses sociales. $»^{19}$ Il s'agit d'une interprétation du concept marxien de fétichisme de la marchandise qui permet de comprendre comment le vécu participe à la valorisation du capital.

Le constat de Vincent, selon lequel les formes de vie s'adaptent aux choses sociales, rappelle l'analyse d'Adorno et Horkheimer, selon laquelle les individus modernes ne disposent plus d'espaces leur permettant de récolter des expériences directes, sous les conditions de ces représentations et médiatisations détournées. L'expérience sensible bute sur des échanges sociaux qui sont réglés par des choses (marchandises, argent, techniques), tandis que la culture et la communication passent par le filtre uniformisant d'une industrie standardisée, l'industrie de la culture.$^{20} \mathrm{En}$ effet, la thèse d'Adorno et Horkheimer au sujet de l'industrie de la culture se réfère directement et explicitement au concept marxien du fétichisme de la marchandise, dans la version originale de la Dialectique de la raison de 1942-44, comme l'a précisé Gérard Raulet. ${ }^{21}$ La référence à Marx n'est gommée ensuite que pour des raisons politiques, par crainte du retournement anticommuniste qui s'opère aux Etats-Unis à cette époque. Dans la Dialectique de la raison, Adorno et Horkheimer nomment la charge régressive de la civilisation occidentale, fondée sur l'autocontrôle et la normalisation de la contrainte, qui est capable de retourner vers la barbarie lorsque son potentiel de violence échappe à la domination ${ }^{22}$. Le concept d'industrie de la culture lie la critique du fétichisme marchand, de la répétition et de la production de stéréotypes qui caractérise le capitalisme, et de l'acculturation.

Chez Adorno, les abstractions sociales qui forment la réalité moderne, le monde administré, la soumission réelle du salariat, l'industrie de la culture et la valorisation marchande de la vie, forment une totalité sociale répressive qui laisse peu de place à une vie individuelle particulière et autonome. Le général prime sur le particulier. La vision d'Adorno trouve ici un terrain de rencontre avec Marx, qui avait insisté sur le fait que la valorisation marchande dépend de la mobilisation subjective des travailleurs payés pour leur capacité à travailler, et non pour le travail accompli. Le degré d'efficacité du travail est, en effet, étroitement lié aux motivations morales, culturelles et symboliques des salariés. L'entreprise est donc autant exposée aux résistances subjectives et révoltes potentielles que l'industrie de la culture, dès que les acteurs se projettent dans le monde à partir de leurs propres interprétations discordantes. La « totalité aveuglante » dont parle Adorno s'avère nettement plus fragile qu'elle ne le paraît au premier regard. Les décalages, discordances historiques et les différences, entre le mouvement général de la société d'un côté, et les vies individuelles de l'autre, rendent à mon sens pensable l'expérience individuelle de la perte des expériences humaines sensibles qui sont désormais recouverts par les abstractions réelles de la société moderne. ${ }^{23}$ À ce titre, le penseur francfortois néglige sans doute le décalage qui existe, entre d'une part l'expérience sensible, et d'autre part la socialisation globale par l'échange abstrait. Même 
si on peut suivre Jean-Marie Vincent quand il indique les correspondances récurrentes entre «la logique de valorisation et les formes de vie », il n'en reste pas moins que cet ensemble vole fréquemment en éclats, grâce aux expériences sensibles des acteurs, éprouvant les contradictions, incohérences et discontinuités des représentations prédominantes. Negt et Kluge ont mis en scène ces explosions révolutionnaires de la modernité dans leur ouvrage Geschichte und Eigensinn, dont la traduction est désormais prévue en France. Loin des apôtres d'une révolution passive, Jean-Marie Vincent proposait de partir de la critique de la vie quotidienne pour parvenir à un changement global: «Les secousses qui ébranlèrent l'ordre social de façon apparemment imprévisible ont une double origine, d'abord la mise en question le plus souvent déstructurée de ce qui se donne pour la "normalité", ensuite la mise à jour de nouvelles formes sociales (communications, institutions embryonnaires, modalités d'action). ». En ce sens, la séparation marxiste classique, entre un champ social et un autre politique, finit par jouer un rôle conservateur en non pas subversif. Le fantasme de tout puissance de la grande politique de la prise du pouvoir ou de l'Etat bloque in fine la capacité inventive des mouvements qui veulent changer le monde. Le marxisme des deux siècles passés, cette pensée-monde, doit renoncer à cette posture pour devenir capable d'accoucher d'une critique neuve. Afin de «devenir au monde ». Rien ne nomme mieux l'enjeu d'une Théorie critique au sein de la crise globale d'un monde finissant.

11 À partir de cette position, une toile conceptuelle se tisse entre Vincent, Gorz, Negri et d'autres penseurs du basculement qui est en train de se produire. Tout en insufflant ce mouvement de débordement conceptuel à partir de Marx, des Grundrisse et de l'Ecole de Francfort, Vincent prend peur devant l'abîme qui s'ouvre devant lui, et qui menace de signaler la fin du mouvement ouvrier en tant que tel. Rien de moins est en jeu dans cette discussion. Gorz autant qu'Habermas reprennent la position d'Arendt, qui avait postulé que ce mouvement s'était épuisé en tant que mouvement de gens libres, en choisissant le champ de la production au détriment de l'espace public, la bureaucratisation à la place d'une politique de la liberté, le faire au lieu de l'agir. Voici le sens caché du sous titre de la Critique du travail. Le faire et l'agir. Gorz tente de trouver l'émancipation en dehors de tout faire, dans le temps libre, les activités non marchandes, la culture non industrielle contre l'industrie de la culture, le règne de la liberté après le règne de la nécessité, en paraphrasant Engels et en se référant aux premières esquisses du Capital, les Grundrisse marxiens. Pourtant, l'abstraction marchande est partout, comme le précise Vincent, la forme valeur doit ainsi être combattue par une action à l'intérieur du faire, contre le faire et au-delà du faire, à la manière du principe autogestionnaire qui voulait déborder le travail pour atteindre la bonne vie. André Gorz apprécie peu cette obstination issue du vingtième siècle, qui enferme la critique dans le champ qui fut autrefois occupé par un marxisme désormais moribond. Il cherche les chemins du paradis, l'autonomie maintenant, partout. Toni Negri, de son côté, au départ aux côtés de Vincent au sein du département de science politique de Vincennes, saisit les Grundrisse de Marx pour imaginer un renversement total, où ceux qui n'ont rien auront tout. L'appauvrissement sous le capitalisme prépare l'éclatement créatif et global d'une multitude planétaire qui saura se reconnaître au milieu de la précarisation généralisée. Vincent pointe la naïveté de la thèse, déjà infirmée du vivant de Marx, mais reste sur la défensive au moment du succès international de Negri, au lieu d'y voir une invitation à relancer la conceptualisation de la soumission du travail vivant au capital mondial, la subsomption réelle. Les deux assauts du ciel, vers le paradis autonome de Gorz et la multitude mondiale de Negri, sont retombés en cendres, alors que Vincent a disparu trop vite. Leur 
idéal subsiste. Les termes de la discussion renaissent aujourd'hui au milieu de la crise mondiale du capitalisme qui fait passer la critique du statut de l'art à la nécessité. Dans son brûlant Crack capitalism, ${ }^{24}$ John Holloway sollicite à nouveau le faire, the doing, pour trouver une source de vitalité qui pourrait briser le poids mortifère des formes capitalistes qui pèsent sur les vivants. Manifestement, il imagine un faire qui serait aussi un agir, mais le concept du faire reste tellement générique que nous ne savons pas où commence la résistance, l'autonomie ou la subjectivité rebelle, et où s'arrête la reproduction des formes sociales dont l'un des stéréotypes est précisément la pseudorébellion et la posture libertaire, comme l'avait vu Adorno. ${ }^{25}$ Lire, revoir et prolonger les débats théoriques fondateurs nous semble une voie prometteuse afin de ne pas revenir sans cesse au même point de départ, ce retour au même schéma, aux impasses politiques du passé et aux apories conceptuelles d'antan. Reprendre le fil rouge afin de surprendre le minotaure.

\section{NOTES}

1. Collection La Quatrième Génération : theoriecritique.com

2. Arno Münster, André Gorz et le socialisme difficile, Lignes, Paris, 2011 ; Stephen Bouquin, «La question des résistances au travail dans la sociologie française ", Actuel Marx n.49, PUF, Paris, 2011 ; Alexander Neumann, «Von der Arbeitskritik zur Multitude» in Kritische Arbeitssoziologie, éd. Schmetterlin, Stuttgart, 2010; Dirk Braunstein, Adornos Kritik der politischen Ökonomie, Transcript Verlag, Bielefeld, 2011.

3. Jean Marie Vincent, «Le mouvement ouvrier en Allemagne de l'ouest de 1945 à 1960. Essai de présentation et d'interprétation. », Doctorat de sciences politiques sous la direction d'Alfred Grosser, Fondation nationale des sciences politiques, Paris, 1962.

4. Adorno, Jargon de l'authenticité, Payot, 1974.

5. Victor Farias, Heidegger et le nazisme, Verdier, 1987 ; Emmanuel Faye, Heidegger, l'introduction du nazisme dans la philosophie, Albin Michel, 2005.

6. Voir aussi Jean Marie Vincent, Fétichisme et société, Anthropos, 1972.

7. Adorno, «Marx und die Grundbegriffe der Soziologie » in : Backhaus, Dialektik der Wertform, ça ira, 1999. Voir aussi Alfred Sohn-Rethel, La pensée marchandise, éd. Du Croquant, Bellecomb, 2009.

8. Axel Honneth, «Arbeit und instrumentales Handeln. Kategoriale Probleme einer kritischen Gesellschaftstheorie » in : Honneth (dir.) Arbeit, Handlung, Normativität, Suhrkamp, Francfort sur le Main, pp.185-233.

9. Honneth, op.cit.

10. Voir la septième édition de Michael Heinrich, Kritik der politischen Ökonomie, Schmettreling, Stuttgart, 2012.

11. Op.cit., p.185.

12. Axel Honneth, Das Recht der Freiheit, Suhrkamp, Berlin, 2011, p.608.

13. Axel Honneth, La société du mépris, La Découverte, Paris, p.199.

14. Ibidem, p.248.

15. Oskar Negt, entretien accordé à la revue Sozialismus, 4/2011, p.56.

16. Adorno, « Marx und die Grundbegriffe der Soziologie » in : Backhaus, Dialektik der Wertform, ça ira, 1997, p.509. 
17. J.M.Vincent, Max Weber ou la démocratie inachevée, Le Félin, 1998, p.80.

18. Backhaus, op.cit., p.18.

19. Vincent, op.cit., p.231.

20. Adorno/Horkheimer, Dialektik der Aufklärung, 1944/1990, p.128.

21. Gérard Raulet, «L'aporie de la théorie critique. Les stratégies de renouvellement du noyau théorique » in : Renault/Sintomer, Où en est la théorie critique ?, La Découverte, Paris, 2003, p.34.

22. Adorno/Horkheimer, Dialectique de la raison, Gallimard, 1990.

23. Alex Demirović, Der non-konformistische Intellektuelle, Suhrkamp, 1999, p.528.

24. John Holloway, Crack capitalism, Pluto Press, 2010; édition française, Libertalia, 2012.

25. Adorno, La personnalité autoritaire, Allia, 2007.

\section{INDEX}

Mots-clés : critique du travail, correspondance, artistes, socialisme vert, travailleurs culturels, Wertkritik, Gorz André, dialogue, Vincent Jean-Marie, écologie, modèles alternatifs, Marx Karl, penser le Capital, ethos du travail, Eglise Orthodoxe, salariat, domination

\section{AUTEUR}

\section{ALEXANDER NEUMANN}

Directeur de publication de Variations. 